\title{
Evaluation of the Procleix Ultrio Elite Assay and the Panther-System for Individual NAT Screening of Blood, Hematopoietic Stem Cell, Tissue and Organ Donors
}

\author{
Albert Heim \\ Institut für Virologie, Medizinische Hochschule Hannover, Hanover, Germany
}

\section{Keywords}

HBV · HCV · HIV · Nucleic acid amplification - Transfusion . Transplantation · Virus safety

\section{Summary}

Background: The performance of the multiplex Procleix Ultrio Elite assay as individual donor nucleic acid test (ID-NAT) for the detection of HIV-1, HIV-2, HCV, and HBV was evaluated in a retrospective, single center study. Methods: ID-NAT results of 21,181 blood donors, 984 tissue donors, 293 hematopoietic stem cell donors and 4 organ donors were reviewed in synopsis with results of serological screening and additional discriminatory and repetitive NAT in case of positive donors. Results: Specificity of the initial Procleix Ultrio Elite assay was $99.98 \%$ and after discriminatory testing $100.00 \%$. Initially invalid results were observed in 75 of 21,181 blood donors $(0.35 \%)$ but 16 of 984 tissue donors $(1.62 \%, p<0.001)$ which included non-heart-beating ('cadaveric') donors. All these had valid negative ID-NAT results after repeated testing or testing of 1:5 diluted specimens in case of tissue donors. Occult hepatitis B (defined here as HBV DNAemia without HBsAg detection) was demonstrated by ID-NAT in two anti-HBc-positive tissue donors and suspected in two other tissue donors, where a definite diagnosis was not achieved due to the insufficient sample volumes available. Conclusion: The Procleix Ultrio Elite assay proved to be specific, robust and rapid. Therefore, routine ID-NAT may also be feasible for organ and granulocyte donors.

(c) 2016 S. Karger GmbH, Freiburg

\section{Introduction}

Routine nucleic acid testing (NAT) of all blood donors for HIV- 1 and HCV is required in Germany in addition to serological screening $[1,2]$. NAT for HIV-2 and HBV is not required in Germany. The risk for HIV-2 transmission during the window period of the serological assay for the detection of antibodies against HIV$1 / 2$ seems to be negligible in Germany because of the low HIV-2 incidence; only about $200 \mathrm{HIV}-2$ infections were reported in the period between 1993 and 2010 [3]. However, the incidence of HIV-2 infections may increase in the future with increasing immigration from Western African countries where HIV-2 is endemic. HBV NAT is not required because of the short diagnostic window period due to $\mathrm{HBsAg}$ testing. Additional anti-HBc screening is required in Germany. In case of HBsAg-negative, but anti-HBc-positive (and anti-HBs $\geq 100 \mathrm{IU} / \mathrm{ml}$ ) blood donors, HBV NAT is required with a LOD (limit of detection; defined as the $95 \%$ probability of detection) of $12 \mathrm{IU} / \mathrm{ml}$ for donor clearance [4].

HIV-1 and HCV NAT is also required for hematopoietic stem cell donors and tissue donors, but not for organ donors (except for organ donors from high-incidence groups for these infections).

NAT of (mini-)pools (MP-NAT) of blood donors instead of NAT of individual donors (ID-NAT) is approvable if a limit of detection of 5,000 IU HCV RNA/ml and 10,000 IU HIV-1 RNA/ml per donor is achieved. MP-NAT is frequently performed in Germany because of limited capacity ('throughput') of in vitro diagnostic systems and with the aim to reduce costs of NAT per donation. However, pooling procedures increase the turnaround time, and in case of a positive result resolution of the pool in order to identify the positive donor (or to distinguish a false-positive result) is tedious and time-consuming. Moreover, ID-NAT holds promise to be more robust compared to MP-NAT in case of mutations at target sites of primers and probes. For example, a MP-NAT was

\section{KARGER \\ Fax +497614520714

\section{() 2016 S. Karger GmbH, Freiburg}

1660-3796/16/0433-0177\$39.50/0 
false-negative due to its very high LOD for the individual donor (caused by a more than 100 -fold higher LOD of the assay due to the mutations and multiplication with a dilution factor according to the pool size) in a case of HIV-1 transmission by blood products of a window phase donor. By contrast, the identical assay performed as ID-NAT would have detected HIV-1 RNA because its increased LOD was still below the virus load of the window phase donor $[5,6]$. Subsequently, 5 other cases of failed HIV-1 RNA detection due to mutations at target sites were described [7,8], and routine double-target NAT for HIV-1 (as in the Ultrio Elite assay) was requested in Germany in order to reduce this risk [7].

ID-NAT for HBV DNA could reduce the risk of HBV transmission both in case of occult hepatitis B (low-level carriers) and during the serological window period $[9,10]$. MP-NAT for HBV DNA is prone to fail in occult hepatitis B due to its higher LOD, and its effect on window period transmissions is small due to the high sensitivity of HBsAg tests $[11,12]$. In a recent study in a low-endemic country (Switzerland), the risk of HBV transmission during the serological window phase was reduced about threefold by ID-NAT, and the only 2 window phase donors detected in this study would have been missed by MP-NAT [10].

The Procleix Ultrio Elite Assay on the Grifols (previously Novartis) Procleix Panther system is CE marked for the multiplex detection of HIV-1, HIV-2, HCV, and HBV nucleic acids in (mini-)pools (up to 96 donors) and individual donor samples, including organ donors and specimens from deceased (non-heart beating) tissue donors. The multiplex assay is based on isothermal transcription-mediated amplification (TMA) instead of the more frequently used polymerase chain reaction (PCR). All steps of the assay, starting with isolation of nucleic acids up to detection of hybridization signals for viral targets and inhibition controls, are performed by the Panther system which operates as an 'order-entry', 'walk away', fully automatic in vitro diagnostic system. The turnaround time from loading a plasma into the Panther system to receiving a result is about $3.5 \mathrm{~h}$ for ID-NAT. The LOD (95\% fiducial limits) for HIV-1, HIV-2, HBV and HCV are 18.0 (15.0-23.5), 10.4 (8.9-12.6), 3.0 (2.5-3.9) and 4.3 (3.8-5.0) IU/ml, respectively. In case of an initially positive result, additional discriminatory assays for HIV-1/2, HCV, and HBV have to be performed for a definite result which requires about another $4 \mathrm{~h}$. Reagents for the discriminatory assays are included in the Ultrio Elite Kit. Both serum and (EDTA-)plasma are appropriate for the Proceix Ultrio Elite Assay. Capacity of a single Panther system (about 70,000 NAT/year) was sufficient for ID-NAT in our setting.

In this study the Procleix Ultrio Elite Assay / Grifols Panther System was evaluated in the setting of a virology department at a university hospital providing services for the screening of blood donors (including a significant proportion of thrombocyte donors), hematopoietic stem cell donors, tissue donors and organ donors. As a short turnaround time was highly advantageous in this setting, routine ID-NAT was performed, which also saved hands on time required for pooling.

\section{Material and Methods}

Donors and Specimens

During the study period (September 2014 to October 2015), 22,462 donors were tested. These included 21,181 blood donors, 984 tissue donors, 293 hematopoietic stem cell donors, and 4 organ donors. In case of blood donors, hematopoietic stem cell donors, and organ donors only plasma was used for testing, whereas in case of tissue donors 656 plasma specimens and 328 serum specimens were tested. 64 tissue donor specimens were sampled up to $12 \mathrm{~h}$ after circulatory arrest, 769 specimens were sampled before circulatory arrest. For 151 tissue donors, no information on sampling before or after circulatory arrest was provided.

\section{Multiplex NAT}

The Procleix Ultrio Elite assay was run on the Panther system as indicated by the manufacturer's instructions. All donor samples were tested by ID-NAT screening with a required sample volume $\geq 0.5 \mathrm{ml}$. 'Cadaveric' samples may be tested diluted $1 / 5$ with $0.9 \% \mathrm{NaCl}$ in order to overcome inhibitors or sample shortage. In case of an initially positive result, the Ultrio Elite discriminatory assays for HIV-1/2, HCV, or HBV were performed. In case of insufficient specimen volume for the three discriminatory assays, a second run of the Ultrio Elite assay was performed.

\section{Screening for Virus Infections}

Abbott Architect Assays for HIV-1/2 (4th generation, antibody and p24 antigen detection), HCV antibodies, HBsAg, Anti-HBc and Treponema pallidum antibodies were performed routinely. In case of donors with positive or equivocal results in serological screening or NAT, testing for HCV antigen, anti-HBs and anti-HBc IgM was also performed on the Abbott Architect platform. Verification of reactive anti $\mathrm{HBc}$ screening results was performed as described previously if a sufficient sample volume was available [4].

\section{Data Mining and Statistical Analysis}

Anonymized results of the Ultrio Elite screening and discriminatory assays were retrieved from the Grifols Panther and cross-checked with the corresponding data set from the laboratory information system using a common identifier (specimen code). Serology results were retrieved from the laboratory information system. Chi-square and Fishers's exact test were performed with help of the Graphpad Prism (version 6) software. For calculating the specificity of the Ultrio Elite Assay, the combined results of the routine serological screening (HIV-1/2, HCV, and HBsAg) were set as a reference.

\section{Results}

\section{General Performance}

22,359 of 22,462 donors had an initially non-reactive result in the Ultrio Elite Assay, and 12 donors had an initially reactive result. An initially invalid result was observed in 75 of 21,181 blood donors $(0.35 \%)$ and in 16 of 984 tissue donors $(1.62 \%, \mathrm{p}<0.001$, Fisher's exact test). By simple re-testing of undiluted specimens in case of blood donors and by testing specimens diluted $1: 5$ with $0.9 \% \mathrm{NaCl}$ in case of tissue donors (in order to dilute potential inhibitors of NAT), valid negative results were generated for all these donors.

Eight of the 12 initially reactive results were confirmed by discriminatory testing and/or concordant positive results of serological screening tests (donors \#2 -7, 10, and 11, for details see below).

Two of the 12 initially reactive results (for blood donor \#1 and tissue donor \#9) had to be revised as negative for HIV-1/2, HCV, and HBV nucleic acids according to the Ultrio Elite test algorithm because discriminatory testing was negative. 
Table 1. Blood donors with initially reactive ID-NAT result

\begin{tabular}{lllllllllllll}
\hline Donor & Ultrio Elite & $\begin{array}{l}\text { Ultrio } \\
\text { Elite } 2^{*}\end{array}$ & $\begin{array}{l}\text { Ultrio } \\
\text { Elite 3* }\end{array}$ & $\begin{array}{l}\text { dHIV } \\
\text { NAT }\end{array}$ & $\begin{array}{l}\text { dHCV } \\
\text { NAT }\end{array}$ & $\begin{array}{l}\text { dHBV } \\
\text { NAT }\end{array}$ & $\begin{array}{l}\text { HIV } \\
\text { Ag/Ab }\end{array}$ & HCV Ab & HBsAg & Anti-HBc & $\begin{array}{c}\text { Anti-HBs } \\
\text { Anti-HBc } \\
\text { IgM }\end{array}$ \\
\hline 1 & + & - & - & - & - & - & - & - & - & - & ND & ND \\
2 & + & + & ND & NV & + & NV & - & + & - & + & - & - \\
3 & + & ND & ND & ND & ND & + & - & - & + & + & - & - \\
\hline
\end{tabular}

${ }^{*}$ First retesting.

${ }^{* *}$ Second retesting.

dHIV, dHCV and dHBV = Discriminatory tests for HIV-1/2, HCV and HBV, respectively (tests provided with the Ultrio Elite Kit); ND = not done;

$\mathrm{NV}=$ no valid result obtained (cross-inhibition of discriminatory assays, because of high HCV DNA concentration).

Table 2. Initially ID-NAT-reactive tissue donors ${ }^{\mathrm{a}}$

\begin{tabular}{|c|c|c|c|c|c|c|c|c|c|c|c|c|}
\hline $\begin{array}{l}\text { Tissue } \\
\text { donor \# }\end{array}$ & Specimen type & Ultrio Elite & $\begin{array}{l}\text { Ultrio } \\
\text { Elite } 2\end{array}$ & dHIV & $\mathrm{dHCV}$ & $\mathrm{dHBV}$ & $\begin{array}{l}\mathrm{HIV} \\
\mathrm{Ag} / \mathrm{Ab}\end{array}$ & $\begin{array}{l}\mathrm{HCV} \\
\mathrm{Ab}\end{array}$ & HBsAg & $\begin{array}{l}\text { Anti- } \\
\mathrm{HBc}\end{array}$ & $\begin{array}{l}\text { Anti-HBs, } \\
\text { IU/l }\end{array}$ & $\begin{array}{l}\text { Anti-HBc } \\
\text { IgM }\end{array}$ \\
\hline 4 & plasma & $+(1: 5)$ & ND & ND & ND & $+(1: 10)^{* *}$ & - & - & - & + & 32 & - \\
\hline 5 & serum & + & ND & ND & ND & $+(1: 4)$ & - & - & - & + & 181 & - \\
\hline 6 & plasma (cadaveric) & + & ND & - & - & + & - & - & + & + & - & - \\
\hline 7 & serum & $+(1: 2)$ & ND & ND & ND & ND & - & - & + & + & ND & - \\
\hline 8 & plasma (cadaveric) & + & + & ND & ND & $-(1: 3)$ & - & - & - & + & 124 & - \\
\hline 9 & plasma & + & ND & - & - & $-(1: 2)$ & - & - & - & + & - & - \\
\hline 10 & serum (cadaveric) & $+($ serum $)$ & $t^{* * *}$ & - & + & - & - & + & - & - & ND & ND \\
\hline 11 & plasma (cadaveric) & + & ND & ND & $+(1: 4)$ & ND & ND & $+^{*}$ & ND & ND & ND & ND \\
\hline 12 & plasma & $+(1: 3)$ & $-(1: 3)$ & ND & $\mathrm{ND}$ & ND & - & - & - & - & ND & ND \\
\hline \multicolumn{13}{|c|}{$\begin{array}{l}{ }^{*} \text { Also positive for HCV antigen. } \\
{ }^{* *} 1: 10 \text { dilution not validated. } \\
{ }^{* *} \text { Additional plasma sample tested. } \\
\text { aSeveral specimens had to be diluted prior to testing due to insufficient volume, dilution factors are indicated in brackets. In donor \#11, serological screening was } \\
\text { performed in another laboratory, merely HCV serology and ID-NAT was requested. } \\
\text { dHIV, dHCV and dHBV = Discriminatory tests for HIV-1/2, HCV and HBV, respectively (tests provided with the Ultrio Elite Kit); ND = not done. }\end{array}$} \\
\hline
\end{tabular}

Three of the 12 initially positive results were not confirmed by discriminatory testing because of insufficient specimen volumes to perform discriminatory testing or other confirmatory testing. Therefore, initial results of these 3 donors were considered to be equivocal. Two of these 3 donors had positive anti-HBc test results (\#8 and \#9), whereas 1 of these 3 donors (\#12) had negative serological screening tests (for details see below).

Counting all 4 initially reactive donors (\#1, 8, 9, and 12), who were not confirmed by discriminatory or confirmatory assays as false-positive, the specificity of the initial Ultrio Eilte run was calculated to be $99.98 \%$. However, only donors with positive Ultrio Elite discriminatory testing should be considered as NAT-positive according to the Ultrio Elite test algorithm. Excluding these donors with an incomplete or negative discriminatory testing, the specificity of the completed Ultrio Elite assay was determined as $100.00 \%$.

\section{Blood Donors}

Detailed results of all blood donors with an initially reactive Ultrio Elite assay $(3$ of 21,181 ) are presented in table 1 . According to the Ultrio Elite test algorithm, donor \# 1 had a negative result in the Ultrio Elite assay because discriminatory testing was negative. In congruence with this decision, the Ultrio Elite(screening) assay was also repeated twice with negative results, and all serological screening tests were also negative. Donor \#2 was found to have a replicative hepatitis $\mathrm{C}$, and a high virus load $\left(1 \times 10^{8} \mathrm{IU} / \mathrm{ml}\right)$ was detected in a subsequent sample by an alternative NAT (Cobas TaqMan). Donor \#3 suffered from a chronic hepatitis B as indicated by the discriminatory HBV NAT and serological test results, but a second sample for virus load testing was not received.

\section{Hematopoietic Stem Cell Donors}

All hematopoietic stem cell donors had negative results in the Ultrio Elite Assay, which was concordant to serological screening results.

\section{Tissue Donors}

Nine of 984 tissue donors had an initially reactive result in the Ultrio Elite assay. Detailed results of all 9 tissue donors with an initially positive Ultrio Elite assay are presented in table 2.

In 4 of these 9 donors, hepatitis B was diagnosed. Occult hepatitis $B$ was diagnosed in 2 donors (\#4 and 5) by detection of HBV DNAemia with help of discriminatory tests and detection of anti$\mathrm{HBc}$ but negative serological tests for HBsAg. Replicative hepatitis B (HBsAg- and anti-HBc-positive) was diagnosed in 2 donors (\#6 
and 7). Two other donors (\#8 and 9) initially positive in the Ultrio Elite assay were also anti-HBc-positive. In donor \#8, the discriminatory assay for HBV DNA was negative but had to be performed with a 1:3 prediluted sample because of an insufficient volume, whereas replicate testing with the Ultrio Elite screening assay was positive. Therefore, a low-level HBV DNAemia with a HBV DNA concentration in the range of (or even below) the LOD of the assay seems to be possible but could not be proven. In donor \#9, discriminatory testing was negative; thus the initially reactive Ultrio Elite result should be reported as negative according to the test algorithm.

In two of the 9 donors (\#10 and 11) a replicative hepatitis $C$ was diagnosed with help of discriminatory testing for HCV RNA. HCV antigen was also detected in donor \#11. These results were in accordance with the detection of antibodies against $\mathrm{HCV}$.

In a single tissue donor (\#12), Ultrio Elite results remained equivocal because discriminatory testing was not feasible due to insufficient specimen volume. In this donor, all serological results were negative, as was a second run of the Ultrio Elite screening assay. Thus, a false-reactive result in the initial Ultrio Elite run can be suspected.

\section{Organ Donors}

All organ donors had negative results in the Ultrio Elite Assay, which was concordant to serological screening results.

\section{Discussion}

A very high specificity of $100.00 \%$ was demonstrated in the present study for 21,476 donors (21,179 blood donors, 293 hematopoietic stem cell donors and 4 heart-beating organ donors) if the complete Ultrio Elite test algorithm was performed (including discriminatory testing). However, discriminatory testing, which delayed the result by about $4 \mathrm{~h}$, was required only in a single blood donor with an initially false-reactive result. This excellent specificity after the initial test run (99.99\%) was slightly better than reported for the older Ultrio assay (99.83\%) with semi-automated handling [13] and for the Ultrio Elite assay (99.95\%) in a multicenter study [14]. Excellent results on specificity and robustness as well as the short turnaround time of the Procleix Ultrio Elite assay / Panther system suggested that ID-NAT seems to be feasible in the future also for granulocyte donors.

Our results on specificity were achieved during the first 13 months of operation of the Ultrio Elite assay at a single site. The Grifols Procleix Panther system was installed in the same room as the Abbott Architect systems, which were used for the serological testing of donors but also for patients of an university hospital, including multiple patients highly positive for HIV-1, HBV, or HCV nucleic acids. Standard operation procedures included precautions to limit the risk of cross-specimen contamination and amplification product contamination. Technicians who switched between work at the Architect and at the Panther had to change gloves and lab coats before working at the Panther. Workflow of donor speci- mens included aliquoting for NAT prior to serological testing. Furthermore, a refrigerator and a desk were reserved for handling of the Ultrio Elite reagents and plastic ware. Cleaning and decontamination procedures were performed as indicated by the manufacturer's instructions. In spite of the excellent specificity of the Ultrio Elite NAT, it cannot completely be excluded that more false-reactive results could be experienced in the future if the laboratory space is contaminated with amplification products e.g. due to failures in decontamination procedures.

In contrast to many other NATs for the detection of HIV-1, $\mathrm{HBV}$ or $\mathrm{HCV}$, the intended use of the Ultrio Elite assay includes the testing of serum specimens and the testing of specimens retrieved from deceased ('non-heart beating', 'cadaveric') donors. Unequivocally positive results were observed in 3 of 328 serum specimens (tissue donors \#5, 7, and 10) and 3 of 63 specimens of 'non-heart beating' tissue donors $(\# 6,10$, and 11). These results clearly supported the claims of the intended use. However, it should be pointed out that the design of the present study does not permit any conclusions on the diagnostic sensitivity of the Ultrio Elite assay because this retrospective evaluation of the routine use of the Ultrio Elite assay did not include the routine testing of all donors with an alternative NAT as a reference. Moreover, the prevalence of positive donors was far too low, notably in the biggest donor groups tested: only 2 of 21,181 blood donors had replicative hepatitis B or hepatitis $\mathrm{C}$, and these were congruently identified by the Ultrio Elite Assay and serological screening tests. Moreover, the anti-HBc prevalence - and hence the risk for occult hepatitis B - was very low, $0.34 \%$ (74 of 21,181$)$ in the blood donor group compared to $5.1 \%$ in the average German population [9]. Therefore, not a single case of occult hepatitis B (low-level HBV DNAemia without HBsAg detection) was found in the blood donor group.

By contrast, anamnestic data on deceased tissue donors is rather incomplete compared to blood donors. Therefore, tissue donors with risk factors for parenterally transmitted virus infections cannot be excluded sufficiently from the tissue donor pool, resulting in a significantly higher prevalence of anti-HBc $(11.7 \%, 115$ of 984) compared to the blood donor group ( $\mathrm{p}<0.0001$, chi square test). Anti-HBc prevalence of tissue donors was even higher than in the average German population (5.1\%) [9]. Two (1.7\%, donors \#4 and 5) of these 115 anti-HBc-positive tissue donors had an occult hepatitis in spite of anti-HBs titers $>10 \mathrm{IU} / \mathrm{ml}$. Two other anti-HBcpositive tissue donors (\#8 and 9) were reactive in the initial multiplex Ultrio Elite screening assay, but discriminatory testing for HBV DNA was negative. Excluding these 2 donors with ambiguous results from calculation, 6 of 984 tissue donors (0.61\%, including 4 donors positive for $\mathrm{HBsAg}$ or $\mathrm{HCV}$ antibodies) were positive for HBV DNA or HCV RNA (significantly more than observed in blood donors ( $\mathrm{p}<0.0001$, Fisher's exact test)).

Discriminatory testing in tissue donors \#8 and 9 had to be performed with diluted samples due to insufficient volumes, which were only available from these donors. Thus results of discriminatory testing may have been false-negative if HBV DNA loads were very low and close to the LOD of the assay. Moreover, the reactive 
result of the initial Ultrio Elite assay was reproduced in donor \#8, supporting the view that this donor was positive for HBV DNA. Therefore, these 2 donors may also be counted as true-positive for HBV DNA, resulting in $0.81 \%$ ID-NAT-positive tissue donors.

A previous study already addressed the problem of initially reactive results, which were negative in (non-diluted) discriminatory testing, using a large group of blood donors tested by the Ultrio and UltrioPlus assay [15]. A clear association with a positive anti$\mathrm{HBc}$ status was found, and about $34 \%$ of these were positive in an alternative HBV NAT or repeated mutiplex Ultrio or Ultrio Plus testing. Therefore, the authors concluded 'that false reactivity cannot be presumed when a donor fails to discriminate' [15]. Two other studies, which compared up to seven different HBV NAT assays, came to a similar conclusion for anti-HBc-positive donors because HBV DNA concentrations can be very low in occult hepatitis $B$ (HBsAg negative, anti-HBc positive) $[16,17]$. Close to the LOD, results of all NAT assays depend on the Poisson distribution, and consequently non-congruent results with replicative testing, discriminatory NAT, and alternative NAT assays (with about similar LODs) can be expected. In spite of this, the application of an ID-NAT with a low LOD should reduce HBV transmission risk [10]. It should be pointed out that LODs for HBV DNA (WHO standard 97/750) are low and almost identical for the Ultrio Elite ID-NAT (4.3 IU/ml, 95\% fiducial limits 3.8-5.0 IU/ml) and the Ultrio Elite discriminatory $\mathrm{HBV}$ assay $(4.5 \mathrm{IU} / \mathrm{ml}, 95 \%$ fiducial limits 4.0-5.3 IU/ml). The low LOD of the Ultrio Elite assay was confirmed $(4.6 \mathrm{IU} / \mathrm{ml}, 95 \%$ fiducial limits $3.8-5.9 \mathrm{IU} / \mathrm{ml})$ by a multicenter performance study [14].

In synopsis, specificity in tissue donors was found to be $100.0 \%$ (976 negative donors had negative results) if the complete Ultrio Elite test algorithm can be performed, but in 3 donors an initially reactive result could not be resolved completely. Either the specimen volume was too small to perform complete confirmatory testing at all $(3 \times 500 \mu \mathrm{l}$ required $)$ or discriminatory testing was only feasible with diluted specimens. In 2 of these 3 donors (\#8 and 9) a true low-level HBV DNAemia seemed to be possible (see above) but in the 3rd tissue donor (\#12), an initially false-reactive result seems to be probable, similar to blood donor \#1. Therefore, the specificity after initial Ultrio Elite multiplex testing of tissue donors may be estimated as $99.9 \%$.

Experiences with tissue donors may be helpful to estimate the potential use of the Ultrio Elite ID-NAT in organ donors. Due to the insufficient number of organ donors and due to time restrictions in testing, loss of organ donors due to delayed or false-positive NAT results is considered to be a major issue. NAT is only required in Germany for organ donors from groups with an increased prevalence of HIV-1/2, HBV, or HCV infections, for example men having sex with men or injecting drug users. Therefore, only 4 organ donors were tested in the Ultrio Elite assay, and all these had negative results. Nevertheless, routine ID-NAT of all organ donors seems to be feasible with the Ultrio Elite Assay because of the short turnaround time (about $3.5 \mathrm{~h}$ ) and the high spec- ificity. Previous American experience with TMA-based NAT (as the Ultrio Elite) indicated already a very high specificity in the organ donation setting [18].

If the experience with 984 tissue donors is used to predict the potential performance of the Ultrio Elite Assay for virtual organ donors, only about $0.1 \%$ of the results would have been delayed by about another $4 \mathrm{~h}$ for discriminatory testing after an initially reactive result. In contrast to non-heart beating tissue donors, discriminatory testing is a feasible strategy in heart-beating organ donors because adequate specimen volumes are usually available.

In about $0.2 \%$ of these virtual organ donors, HBV DNAemia would have been detected (or probably detected) in spite of high anti-HBs titers (see anti-HBc-positive tissue donors \#5 and 8). However, allocation of organs of anti-HBc-positive organ donors is already restricted (e.g. to HBsAg-positive recipients) if anti-HBs titers are low or negative but anti-HBc-positive organ donors are not rejected in general. Therefore, the additional loss of organs due to NAT positive for HBV seems to be negligible, whereas virus safety should be increased, e.g. due to detection of HCV window period infections. Whether or not the detection of HBV DNA was really associated with infectivity of organs (other than the liver) cannot be resolved in the 2 cases mentioned above. In general, high titers of anti-HBs should neutralize infectious virus but HBV variants with immune escape mutations may still be infectious [19]. On the other hand, it may be speculated that the detected HBV DNA was perhaps not associated with virus particles but that HBV covalently closed circuit DNA may be released from liver cells during terminal states of circulatory shock prior to death when blood sampling may have been performed in these tissue donors.

In spite of these unresolved issues on HBV DNA detection in anti-HBc-positive donors, ID-NAT should clearly improve virus safety of organ donors because of the long window period of $\mathrm{HCV}$ antibody tests, as it was described for blood donors previously [20]. Due to the short doubling time of HCV RNA loads in peripheral blood, a 1:5 dilution, which may be required for specimens containing inhibitors, will not compromise the performance of $\mathrm{HCV}$ NAT [20].

In conclusion, the Procleix Ultrio Elite assay / Panther system proved to be rapid, highly specific, and robust. Thus, routine IDNAT of organ donors and granulocyte donors seems to be feasible in future.

\section{Acknowledgement}

The author wishes to thank Ruth von Piotrowski and Beate Klose for excellent technical assistance.

\section{Disclosure Statement}

The author received a travel grant and speaker's honorarium from Grifols, Germany. 


\section{References}

1 Bekanntmachung über die Ergebnisse des Stufenplanverfahrens zur Verminderung des Risikos von Hepatitis B -, Hepatitis C- und HIV-Infektionen bei Empfängern von Erythrozytenkonzentraten vom 25.Februar 1998. Bundesanzeiger 1998;53:3835.

2 Verminderung des Risikos von HIV-1-Infektionen durch zelluläre Blutprodukte und Gefrorenes Frischplasma Anordnung der Testung auf HIV-1-RNA mit Nukleinsäure-Amplifikationstechniken (vom 06. Mai 2003). Bundesanzeiger 2003;103:12269.

3 RKI: HIV-Infektionen und AIDS-Erkrankungen in Deutschland 2009. Epid Bull 2010;22:205-220 (last accessed April 18, 2016).

4 Mitteilungen des Arbeitskreises Blut des Bundesministeriums für Gesundheit: Aktualisierung der Voten 34 und 35 «Verfahren zur Rückverfolgung (Look Back) (gemäß \$ 19 Transfusionsgesetz)» vom 14.06.2006 im Hinblick auf Hepatitis-B-Infektionen. Bundesgesundheitsblatt Gesundheitsforschung Gesundheitsschutz 2013;56:476-478.

5 Korn K, Weissbrich B, Henke-Gendo C, Heim A, Jauer CM, Taylor N, Eberle J: Single-point mutations causing more than 100-fold underestimation of human immunodeficiency virus type 1 (HIV-1) load with the cobas taqman HIV-1 real-time PCR assay. J Clin Microbiol 2009;47:1238-1240.

6 Schmidt M, Korn K, Nubling CM, Chudy M, Kress J, Horst HA, Geusendam G, Hennig H, Sireis W, Rabenau HF, Doerr HW, Berger A, Hourfar MK, Gubbe K, Karl A, Fickenscher H, Tischer BK, Babiel R, Seifried E, Gürtler L: First transmission of human immunodeficiency virus type 1 by a cellular blood product after mandatory nucleic acid screening in Germany. Transfusion 2009;49:1836-1844.
Chudy M, Kress J, Halbauer J, Heiden M, Funk MB, Nübling CM: Risk minimization measures for blood screening HIV-1 nucleic acid amplification technique assays in Germany. Transfus Med Hemother 2014;41:45-51.

8 Chudy M, Weber-Schehl M, Pichl L, Jork C, Kress J, Heiden M, Funk MB, Nübling CM: Blood screening nucleic acid amplification tests for human immunodeficiency virus type 1 may require two different amplification targets. Transfusion 2012;52:431-439.

-9 Poethko-Müller C, Zimmermann R, Hamouda O, Faber M, Stark K, Ross RS, Thamm M: Die Seroepidemiologie der Hepatitis A, B und C in Deutschland. Ergebnisse der Studie zur Gesundheit Erwachsener in Deutschland (DEGS1). Bundesgesundheitsblatt Gesundheitsforschung Gesundheitsschutz 2013;56:707-715

10 Stolz M, Tinguely C, Graziani M, Fontana S, Gowland P, Buser A, Michel M, Canellini G, Zuger M, Schumacher P, Lelie N, Niederhauser C: Efficacy of individual nucleic acid amplification testing in reducing the risk of transfusion-transmitted hepatitis B virus infection in Switzerland, a low-endemic region. Transfusion 2010;50:2695-2706.

11 Laperche S: Blood safety and nucleic acid testing in Europe. Euro Surveill 2005;10:3-4.

12 Weusten J, Vermeulen M, van Drimmelen H, Lelie N: Refinement of a viral transmission risk model for blood donations in seroconversion window phase screened by nucleic acid testing in different pool sizes and repeat test algorithms. Transfusion 2011;51:203-215.

13 Koppelman MH, Assal A, Chudy M, Torres P, de Villaescusa RG, Reesink HW, Lelie PN, Cuypers HT: Multicenter performance evaluation of a transcriptionmediated amplification assay for screening of human immunodeficiency virus-1 RNA, hepatitis $\mathrm{C}$ virus
RNA, and hepatitis B virus DNA in blood donations. Transfusion 2005;45:1258-1266.

14 Grabarczyk P, Koppelman M, Boland F, Sauleda S, Fabra C, Cambie G, Kopacz A, O’Riordan K, van Drimmelen H, O'Riordan J, Lelie N: Inclusion of human immunodeficiency virus type 2 (HIV-2) in a multiplex transcription-mediated amplification assay does not affect detection of HIV-1 and hepatitis B and $\mathrm{C}$ virus genotypes: a multicenter performance evaluation study. Transfusion 2015;55:2246-2255.

5 Charlewood R, Flanagan P: Ultrio and Ultrio plus nondiscriminating reactives: false reactives or not? Vox Sang 2013;104:7-11.

16 Dettori S, Candido A, Kondili LA, Chionne P, Taffon S, Genovese D, Iudicone P, Miceli M, Rapicetta M: Identification of low HBV-DNA levels by nucleic acid amplification test (NAT) in blood donors. J Infect 2009;59:128-133.

17 Enjalbert F, Krysztof DE, Candotti D, Allain JP, Stramer SL: Comparison of seven hepatitis B virus (HBV) nucleic acid testing assays in selected samples with discrepant HBV marker results from United States blood donors. Transfusion 2014;54:2485-2495.

18 Mone T: History and lessons learned from NAT testing on the west coast. 2012. www.onelegacy.org/site/ docs/TMA_NAT00_Mone_HistoryAndLessonsLearned_ 2012.pdf (last accessed April 18, 2016)

19 Besharat S, Katoonizadeh A, Moradi A: Potential mutations associated with occult hepatitis B virus status. Hepat Mon 2014;14:e15275.

20 Nübling CM, Unger G, Chudy M, Raia S, Lower J: Sensitivity of HCV core antigen and HCV RNA detection in the early infection phase. Transfusion 2002;42: 1037-1045. 